Bio - grafia. Escritos sobre la Biología y su Enseñanza. ISSN 2027

Edición Extraordinaria. p.p. 1424-1435

Memorias del VIII Encuentro Nacional de Experiencias en Enseñanza de la Biología y la Educación Ambiental. III Congreso Nacional de Investigación en Enseñanza de la Biología.

\title{
Análisis del discurso escrito de estudiantes universitarios acerca de la inmunidad
}

López, Ana Milena; ; Orrego, Mary y Tamayo, Óscar Eugenio ${ }^{3}$

\section{Resumen}

Se realizó un estudio comprensivo sobre las características del discurso escrito de 37 estudiantes universitarios, acerca de los procesos que se desencadenan en el sistema inmune como respuesta a la acción de microagresores. Para la recolección de la información se aplicó un cuestionario tipo Likert y preguntas abiertas sobre casos clínicos. A partir del análisis de las oraciones con sentido u oraciones nucleares (Chomsky, 2004) dadas por los estudiantes sobre la inmunidad, ubicamos los textos en tres modelos explicativos, relacionados con la historia de la inmunología y de allí caracterizamos el discurso escrito de los estudiantes a partir de dos categorías: coherencia y estructura discursiva.

Dentro de los principales resultados se destaca la elaboración de textos con coherencia local y el uso de conectores causales, tanto en expresiones cortas como en los textos más largos. Asimismo, es frecuente el uso de lenguajes tautológicos, que reflejan poca comprensión y evidencian el empleo de discursos descriptivos, los cuales pueden constituirse en un obstáculo para el aprendizaje.

Palabras clave: inmunidad, discurso, causalidad, modelos explicativos.

Title: Written Discourse Analysis of the University Students about Immunity

\section{Abstract}

\footnotetext{
${ }_{1}^{1}$ Coordinadora académica Corporación Parque Explora. cpe.analopez@gmail.com.

2 Profesora en Universidad Autónoma de Manizales y Universidad Nacional de Colombia sede Manizales. maryorrego@gmail.com.

3 Vicerrector Académico Universidad de Caldas. Profesor Universidad Autónoma de Manizales. oscar.tamayo@ucaldas.edu.co.
} 
Bio - grafia. Escritos sobre la Biología y su Enseñanza. ISSN 2027

Edición Extraordinaria. p.p. 1424-1435

Memorias del VIII Encuentro Nacional de Experiencias en Enseñanza de la Biología y la Educación Ambiental. III Congreso Nacional de Investigación en Enseñanza de la Biología.

A comprehensive study on written discourse was performed on 37 university students, about the processes that are triggered in the immune system as a response to the action of micro attackers. For gathering this information, the university students were asked to respond a Likert questionnaire and open-ended questions about clinical cases. From the analysis of the sense sentences or nuclear sentences (Chomsky, 2004) used by the students about immunity, we classified the texts into three explicative models related to the history of immunology and then we proceed to characterize the written discourse of the students based on two categories: coherence and discursive structure.

Among the main results are the emphasis on the construction of texts with local coherence and the use of causal connectors, both in short expressions and long texts. In the same way the use of tautological speech is frequent, which reflects little comprehension and reveals the use of a descriptive discourse that might result into a learning impediment

Keywords: immunity, discourse, causality, explicative models.

\section{Introducción}

El análisis del discurso se ha convertido en una herramienta que permite entender no solo el lenguaje, las palabras, sino también la retórica, las ideas, los saberes, la experiencia y la influencia de los contextos. En ese sentido, el lenguaje, dentro de una comunidad determinada, además de vocabulario y gramática es un sistema de recursos para crear significados, es una semántica (Luria 1984, Vygotsky 1995, Lemke 1997, Sutton 1997, citados por Tamayo y Sanmartí, 2005).

Aunque los estudios del análisis del discurso, iniciaron en el seno de la lingüística, se han convertido en la actualidad en un punto de partida para comprender la influencia del contexto y los aprendizajes culturales en la adquisición de conceptos científicos. La importancia que en la actualidad tienen los estudios acerca del lenguaje para la Educación

en Ciencias reside, entre otros aspectos, en sus funciones como vía comunicativa privilegiada en la enseñanza y en el aprendizaje y como mediador y regulador del desarrollo del pensamiento de los estudiantes (Tamayo y Sanmartí, 2005). 
Bio - grafia. Escritos sobre la Biología y su Enseñanza. ISSN 2027

Edición Extraordinaria. p.p. 1424-1435

Memorias del VIII Encuentro Nacional de Experiencias en Enseñanza de la Biología y la Educación Ambiental. III Congreso Nacional de Investigación en Enseñanza de la Biología.

El análisis de los textos escritos puede realizarse desde su estructura y funcionalidad. Al respecto, Tamayo (2001), manifiesta que la estructura tiene que ver con las palabras, proposiciones y oraciones - con base en reglas lógicas - que constituyen un discurso. Aquí, juega un papel importante el uso generalizado de conectores causales junto con el tipo de explicación empleado por los estudiantes, aunque se reconoce que en un discurso escrito sus oraciones no están solo unidas por conectores lógicos.

En el análisis del discurso, cobra importancia la coherencia y estructura discursiva. La coherencia de un discurso puede ser local o global. Teun van Dijk (1992) establece que la coherencia lineal o local se define en términos de las relaciones semánticas entre oraciones individuales de la secuencia; es decir, la relación que existe entre las oraciones. La coherencia global se refiere a como la idea central toma sentido, cuando sus distintas partes mantienen relaciones de significado.

Abre, Maximo, Cremonini y Coutinho (2015) establecen que para enseñar en el contexto universitario, se deben tener en cuenta los conocimientos previos de los futuros profesionales, pero no existe ninguna mención al reconocimiento ni la importancia de esto en el área de la salud y menos en temas relacionados con la inmunidad. Asimismo, estos autores establecen que la enseñanza de la respuesta inmune ocurre de forma aislada, lo que parece paradójico, pues los estudiantes no logran vincular sus conocimientos con la vida cotidiana.

\section{Metodología}

La investigación se realizó con 37 estudiantes de segundo semestre de un área de la salud. Se realizó un estudio descriptivo- comprensivo, en el que se ubicaron las respuestas de los estudiantes universitarios en los distintos modelos explicativos encontrados en la historia de la inmunología y a partir de allí, se describió la coherencia y la estructura del discurso escrito de los estudiantes. Para la recolección de la información se aplicó un cuestionario tipo Likert y se incluyeron además, preguntas abiertas referidas a situaciones cotidianas o casos clínicos relacionados con los mecanismos de respuesta del sistema inmune (ver tabla 1). 
Bio - grafia. Escritos sobre la Biología y su Enseñanza. ISSN 2027

Edición Extraordinaria. p.p. 1424-1435

Memorias del VIII Encuentro Nacional de Experiencias en Enseñanza de la Biología y la Educación Ambiental. III Congreso Nacional de Investigación en Enseñanza de la Biología.

Tabla 1 - Ejemplo de preguntas $(P)$ empleadas para la recolección de la información.

Tipología de preguntas realizadas para la recolección de la información

1. A los estudiantes de las facultades de salud se les exige al ingreso el certificado de la vacuna contra la hepatitis B. ¿Puedes explicar por qué sucede esto?

16. Las personas de raza negra son más susceptibles a sufrir tuberculosis que las personas de raza blanca.

CA A D CD Porque:

Para el análisis de la información, se identificaron oraciones nucleares o con sentido (Chomsky, 2004) dadas por los estudiantes acerca de la inmunidad y de allí se ubicaron en los modelos explicativos o conceptuales de la inmunología, encontrados en la literatura. Posteriormente, se seleccionaron los textos más largos dentro de cada modelo explicativo y se determinó el tipo de discurso empleado, teniendo en cuenta la coherencia, la estructura, la exigencia conceptual y el sentido de cada idea en la inmunidad.

\section{Resultados y discusión}

Para el análisis de la información se obtuvieron 720 oraciones con sentido, otras respuestas fueron descartadas, pues no aportaban ninguna información relevante para analizar. A continuación se presentan los resultados de la investigación.

\section{Modelos explicativos}

El desarrollo histórico de la inmunidad se ha sintetizado en seis modelos explicativos, que corresponden a los distintos momentos en los que ha ido cambiando el concepto a lo largo de la historia.

A continuación se presenta el análisis de las oraciones nucleares dadas por los estudiantes y su ubicación en los diferentes modelos explicativos:

El $36 \%$ de las expresiones analizadas se ubican dentro del modelo clásico, en el cual la respuesta inmune tiene su fundamento en una analogía de tipo militar, donde el funcionamiento del sistema inmune se activa frente a la amenaza proveniente "del exterior, 
Bio - grafia. Escritos sobre la Biología y su Enseñanza. ISSN 2027

Edición Extraordinaria. p.p. 1424-1435

Memorias del VIII Encuentro Nacional de Experiencias en Enseñanza de la Biología y la Educación Ambiental. III Congreso Nacional de Investigación en Enseñanza de la Biología.

percibida como externa" (Varela, 1997). En este tipo de respuestas, los estudiantes usan términos como atacar, combatir y batallar.

Dentro del modelo pre-científico se destaca el postulado de Tucídides "no se sufre la enfermedad dos veces y de padecerla, la recaída nunca es mortal" (Iglesias-Gamarra, Siachoque, Pons-Estel, Restrepo, Quintana y Gómez, 2009). El 12\% de las oraciones se ubican en este modelo.

El $47 \%$ de las respuestas analizadas se ubican en el modelo científico, en el cual se identifica un lenguaje adecuado y conceptos propios de la inmunidad; sin embargo, las explicaciones son superficiales y se desconocen los mecanismos moleculares que suceden al interior de las células.

El $5 \%$ de las explicaciones no se ubican en ningún modelo, pues se basan en la observación y la experiencia personal.

\section{Coherencia del discurso y exigencia conceptual}

La coherencia de un discurso la determina el orden de las oraciones y su significado en un contexto determinado: puede ser global o local. En el análisis del discurso de los estudiantes, la coherencia local y global determinan, en unión con el contexto en el cual se genera (para este caso la inmunidad), sus múltiples significados (Tamayo, 2001). En esta categoría, el análisis se realizó a partir de los textos más largos elaborados por los estudiantes dentro de cada uno de los modelos encontrados anteriormente.

\section{Modelo explicativo clásico}

Se presenta a continuación una respuesta de los estudiantes y su respectivo análisis:

P10.E25. "1[se forman debido a los leucocitos que llegan atacar la infección en las amígdalas].

2[Los leucocitos buscan atacar los microorganismos que causan la infección], 3[los cuales causan a su vez la amigdalitis]." 
Bio - grafia. Escritos sobre la Biología y su Enseñanza. ISSN 2027

Edición Extraordinaria. p.p. 1424-1435

Memorias del VIII Encuentro Nacional de Experiencias en Enseñanza de la Biología y la Educación Ambiental. III Congreso Nacional de Investigación en Enseñanza de la Biología.

El texto es claramente tautológico, pues las ideas 1 y 2 son iguales - los leucocitos atacan la infección- y con la idea 3 concluye que los microorganismos causan la infección. En la tabla 2 se relaciona el número de ideas con su significado para la inmunidad:

Tabla 2- Número de ideas del texto P10.E25., y su significado en la inmunidad.

\begin{tabular}{|c|l|}
\hline \multicolumn{1}{|l|}{ Idea } & Significado desde la inmunidad \\
\hline 1 & Reconocimiento de lo extraño \\
\hline 2 & Reconocimiento de lo extraño \\
\hline 3 & Enfermedad infecciosa \\
\hline
\end{tabular}

Se observa que es un enunciado superficial, redundante y aunque utiliza conceptos propios de la inmunología, desconoce los procesos que suceden a nivel molecular. Dentro del modelo explicativo clásico se encuentran discursos con coherencia local. Se destaca el hecho de sólo haber encontrado un máximo de 40 palabras (P14.E32.) y máximo 4 ideas para explicar los fenómenos relacionados con la inmunidad, lo que refleja discursos poco reflexivos, con coherencia lineal, poco elaborados, con poca exigencia conceptual y pocas ideas alejadas de la pregunta (tautológicos).

\section{Modelo explicativo pre-científico}

El modelo pre-científico se relaciona con el postulado de Tucídides, quien estableció que algunas enfermedades solo se padecen una vez en la vida y que de padecerla, la recaída nunca es mortal. En este ámbito se ubica la siguiente respuesta:

P14.E3. "CA, 1 [porque la primera vez que se presenta esta enfermedad el organismo crea una inmunidad] 2[y por lo tanto cuando vuelve a aparecer el organismo ya está preparado] 3[y sabe cómo defenderse]."

En la tabla 3 se presentan las ideas descritas en el texto P14.E3., y su significado en la inmunidad: 
Bio - grafia. Escritos sobre la Biología y su Enseñanza. ISSN 2027

Edición Extraordinaria. p.p. 1424-1435

Memorias del VIII Encuentro Nacional de Experiencias en Enseñanza de la Biología y la Educación Ambiental. III Congreso Nacional de Investigación en Enseñanza de la Biología.

Tabla 3- Número de ideas del texto P14.E3., y su significado desde la inmunidad.

\begin{tabular}{|c|l|}
\hline Idea & \multicolumn{1}{|c|}{ Significado desde la inmunidad } \\
\hline 1 & $\begin{array}{l}\text { Exposición a antígenos - inmunidad } \\
\text { adquirida }\end{array}$ \\
\hline 2 & Producción de anticuerpos \\
\hline 3 & Respuesta inmunológica \\
\hline
\end{tabular}

Se evidencia coherencia local, discurso causal y condicional, en el que el estudiante trata de dar a entender que la exposición a un agente patógeno por primera vez, conlleva a una inmunidad adquirida que induce memoria inmunológica; si el patógeno regresa nuevamente se produce una respuesta inmunológica y no se manifiestan síntomas clínicos.

El análisis de este texto muestra que el estudiante utiliza explicaciones cercanas a concepciones sociales, donde se sabe que la varicela, por ejemplo, solo aparece una vez en la vida, pero no se hace uso de conceptos científicos. Esto significa que su discurso se limita a la reproducción de ideas inmersas en el ámbito cultural y de lo que encuentra en la pregunta.

\section{Modelo explicativo científico}

El modelo científico tiene unas características que lo diferencian de los otros: vacunación, se comprende que las bacterias son causantes de las enfermedades, se habla de inflamación y fagocitosis como mecanismos inmunológicos, entre otros. A continuación se analiza la coherencia discursiva de una respuesta que se ubica en el modelo científico:

P19.E26. "CA, 1 [las zonas tropicales y húmedas habitan muchos mosquitos y zancudos y hay mucha humedad que es la vivienda de estos animalitos] 2[y están más en contacto con nosotros] 3[y al picarnos nos puede producir esta enfermedad] 4[pero si poseemos esta vacuna] 5[estos anticuerpos actúan cuando vean microorganismos desconocidos en nuestro cuerpo]." 
Bio - grafia. Escritos sobre la Biología y su Enseñanza. ISSN 2027

Edición Extraordinaria. p.p. 1424-1435

Memorias del VIII Encuentro Nacional de Experiencias en Enseñanza de la Biología y la Educación Ambiental. III Congreso Nacional de Investigación en Enseñanza de la Biología.

En este caso, se encuentran 5 ideas que se mezclan, dificultando la identificación de ideas unificadoras; es decir, es un texto poco coherente, con ausencia de algunos conectores que faciliten su lectura y comprensión. En otras palabras, su proceso de generalización no le ha permitido identificar las ideas esenciales desde las cuales organizar su discurso, o lo que es lo mismo, su proceso de generalización es tan vago que no es posible identificar algunas ideas como centrales que le permitan elaborar discursos con coherencia global (Tamayo y Sanmartí, 2005)

En la tabla 4 se muestran las ideas identificadas en el texto P19.E26., y el significado que éstas tienen en la inmunidad:

Tabla 4 - Número de ideas del texto P19.E26., y su significado desde la inmunidad.

\begin{tabular}{|c|l|}
\hline Idea & \multicolumn{1}{|c|}{ Significado desde la inmunidad } \\
\hline 1 & Hábitat \\
\hline 2 & Interacción parásito hospedero \\
\hline 3 & Etiología de la enfermedad \\
\hline 4 & Inmunidad adquirida - vacunación \\
\hline 5 & Anticuerpos - respuesta inmunológica \\
\hline
\end{tabular}

Teniendo en cuenta el contexto del enunciado y la respuesta de este estudiante, se considera que la idea 1 es bastante confusa pues cuando dice "animalitos" no es claro si se refiera al virus que causa la malaria o a los mosquitos - vectores biológicos del virus - y zancudos de los que venía hablando. Cuando pasa a la idea 2 no encuentra una oración que permita enlazar las ideas 1 y 2 claramente. Con la idea 3 , este estudiante explica que los mosquitos o zancudos cuando pican producen la enfermedad; parece que no comprende que es el mosquito el que transmite, pero no la enfermedad, sino el virus. Posteriormente utiliza un adversativo - con la idea 4 - afirmando que algo sucede si poseemos la vacuna.

Este estudiante, a pesar de ubicarse en el modelo explicativo científico, no se exige conceptualmente y pareciera que durante su explicación pusiera en juego más su percepción sobre los eventos estudiados que los conocimientos aprendidos. 
Edición Extraordinaria. p.p. 1424-1435

Memorias del VIII Encuentro Nacional de Experiencias en Enseñanza de la Biología y la Educación Ambiental. III Congreso Nacional de Investigación en Enseñanza de la Biología.

\section{Estructura del discurso}

Para comprender la estructura del discurso se tuvo en cuenta el tipo de conectores que utilizan los estudiantes en sus respuestas. Los conectores utilizados se agruparon en tres categorías: los causales que incluye los conectores de causa, de condición y de consecuencia; los de certeza o de razón y los de oposición.

El $94 \%$ de las explicaciones dadas por los estudiantes, emplean conectores causales. De este total, el $78 \%$ utilizan conectores de causa, el $12 \%$ de condición y el $10 \%$ de consecuencia. El restante lo constituyen $4 \%$ de conectores de certeza y $2 \%$ de oposición. Para un mejor análisis se presenta a continuación la siguiente tabla (ver tabla 5):

Tabla 5 - Tipo de conectores empleado por los estudiantes en su discurso acerca de la inmunidad.

\begin{tabular}{|c|c|c|c|c|c|c|}
\hline \multirow[b]{2}{*}{ Texto } & \multirow[b]{2}{*}{ Oraciones } & \multicolumn{5}{|c|}{ Conectores } \\
\hline & & ca & d & co & ra & op \\
\hline $\begin{array}{l}\text { "Porque al tener amigdalitis las } \\
\text { amígdalas tienen un poco de } \\
\text { hinchazón y al consumir algún } \\
\text { alimento afecta las amígdalas } \\
\text { ye se producen placas } \\
\text { blanquecinas." (P10.E28.) }\end{array}$ & $\begin{array}{l}\text { Porque al tener amigdalitis } \\
\text { las amígdalas tienen un } \\
\text { poco de hinchazón y al } \\
\text { consumir algún alimento } \\
\text { afecta las amígdalas y se } \\
\text { producen placas } \\
\text { blanquecinas." }\end{array}$ & $x$ & & $x$ & & \\
\hline
\end{tabular}


Bio - grafia. Escritos sobre la Biología y su Enseñanza. ISSN 2027

Edición Extraordinaria. p.p. 1424-1435

Memorias del VIII Encuentro Nacional de Experiencias en Enseñanza de la Biología y la Educación Ambiental. III Congreso Nacional de Investigación en Enseñanza de la Biología.

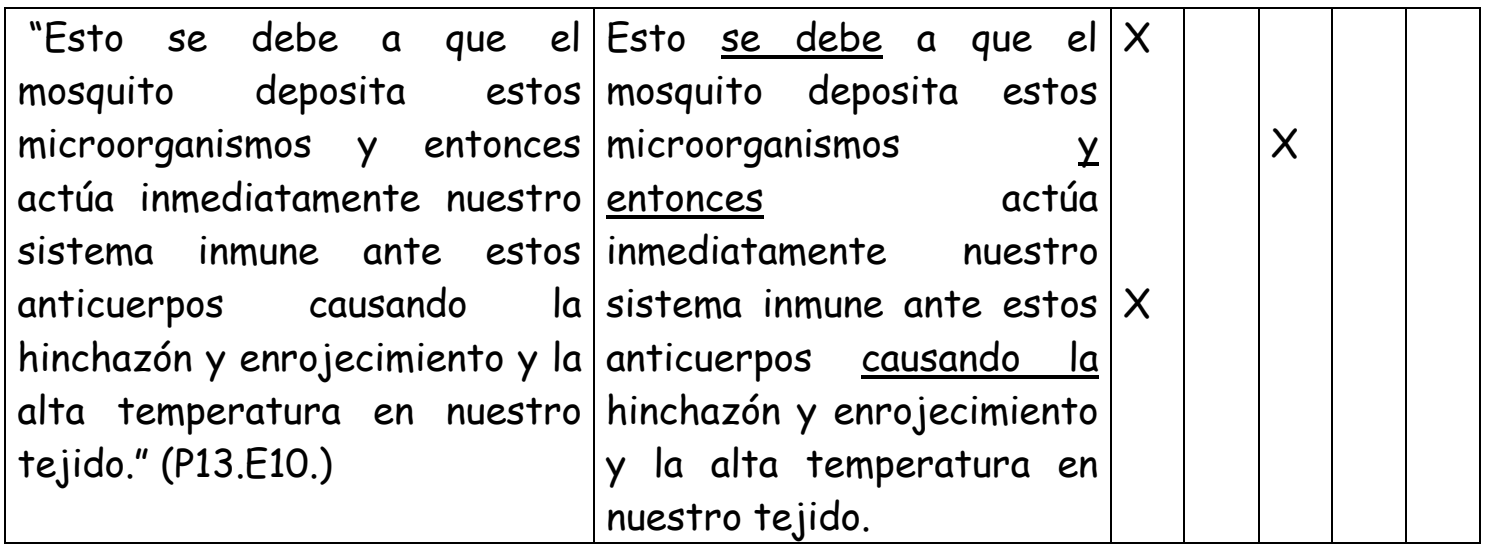

Los textos presentados emplean conectores causales, ya sean estos de causa, consecuencia o condición. Este alto uso de conectores causales tanto en expresiones cortas como en textos largos y, la organización de las proposiciones de manera lineal, hace pensar en el empleo del pensamiento causal por parte de los estudiantes.

\section{Conclusiones}

El análisis sugiere que los textos de los estudiantes no presentan coherencia global, pues se limitan en muchas ocasiones a responder con el texto base sin poner en juego otras explicaciones y hacer alusión a otros conceptos de la inmunología. El tipo de causalidad empleada en el discurso de los estudiantes es simple; es evidente que utilizan pocas variables y de manera lineal. Se entiende que esta causalidad simple (lineal) está determinada por la sucesión plana de causas y de efectos.

Se observa la tendencia general a elaborar discursos donde no es clara la funcionalidad de las diferentes proposiciones utilizadas en el conjunto total de la explicación. Esta baja coherencia funcional está acompañada, en general, con el uso de un bajo número de proposiciones diferentes, (bajo número de variables en la explicación). Además, sobre la exigencia conceptual, se estableció que es muy baja y, mucho más para el contexto universitario, donde se supone que los estudiantes deben un mejor dominio conceptual. Esto sugiere entonces, un aprendizaje superficial. 
Bio - grafia. Escritos sobre la Biología y su Enseñanza. ISSN 2027

Edición Extraordinaria. p.p. 1424-1435

Memorias del VIII Encuentro Nacional de Experiencias en Enseñanza de la Biología y la Educación Ambiental. III Congreso Nacional de Investigación en Enseñanza de la Biología.

Conocer los usos discursivos de los estudiantes tiene propósitos de diferente naturaleza. En primer lugar aporta acerca de la incorporación del lenguaje y de la semiótica en los procesos de enseñanza y aprendizaje de las ciencias, en el marco de los trabajos ya clásicos de Lemke en los cuales se destaca el valor del lenguaje en la enseñanza y aprendizaje. En segundo lugar, en la medida en que permite refinar procesos metodológicos en los que se usen el análisis del discurso y del contenido en función de comprender situaciones de aula. En tercer lugar, y seguramente la de mayor significado en esta investigación, referida de manera específica al valor y necesidad de conocer en detalle aspectos cognitivo-linguísticos de los estudiantes, identificar cómo éstos se pueden constituir en posibles obstáculos frente al aprendizaje de las ciencias y, en consecuencia, orientar acciones de enseñanza en función de superar tales obstáculos. Vinculado con lo anterior, se destaca la necesidad de reconocer diferentes tipologías de estudiantes en cuanto a sus usos del lenguaje en el aula, con el propósito de orientar acciones de enseñanza diferenciadas que posibiliten el avance de ellos en el marco de los objetivos propuestos.

\section{Referencias bibliográficas}

Abreu, V., Maximo, M., Cremonini, T. y Coutinho, R. (2015). El uso de un organizador previo en la enseñanza de Inmunología. Revista Eureka sobre Enseñanza y Divulgación de las Ciencias 12(1), 38-54.

Chomsky, N. (2004). Estructuras sintácticas. México: Siglo Veintiuno.

Iglesias-Gamarra, A., Siachoque, H., Pons-Estel, B., Restrepo, J.F., Quintana L.G. y Gómez, G.A. (2009). Historia de la autoinmunidad. Primera Parte, La inmunología ¿desde dónde y hacia dónde? Revista Colombiana de Reumatología, 1 (16), 11-31.

Tamayo, Ó.E. (2001). Evolución conceptual desde una perspectiva multidimensional. Aplicación al concepto de respiración (Tesis doctoral). Universidad Autónoma de Barcelona, Barcelona. 
Bio - grafia. Escritos sobre la Biología y su Enseñanza. ISSN 2027

Edición Extraordinaria. p.p. 1424-1435

Memorias del VIII Encuentro Nacional de Experiencias en Enseñanza de la Biología y la Educación Ambiental. III Congreso Nacional de Investigación en Enseñanza de la Biología.

Tamayo, Ó.E. y Sanmartí, N. (2005). Características del discurso escrito de los estudiantes en clase de ciencias. Revista Latinoamericana de Ciencias Sociales, Niñez y Juventud, vol. 3 , n. 2, p. 2- 20.

Varela, F. (1997). El Segundo cerebro del cuerpo en: Fischer, H. R., Retzer, A., Schweitzer, J., \& Congreso Interdisciplinario: "El final de los grandes proyectos y el florecimiento de la praxis sistémica" (1991: Heidelberg). El final de los grandes proyectos. Editorial Gedisa, 107- 113 .

van Dijk, T. (1992). La ciencia del texto. Barcelona: Editorial Paidós. 